\title{
Verbascoside Inhibits Glioblastoma Cell Proliferation, Migration and Invasion While Promoting Apoptosis Through Upregulation of Protein Tyrosine Phosphatase SHP-1 and Inhibition of STAT3 Phosphorylation
}

\author{
Wei-Qiang Jia ${ }^{a}$ Zhao-Tao Wang ${ }^{a}$ Ming-Ming Zou ${ }^{a}$ Jian-Hao Lin ${ }^{b}$ \\ Ye-Hai Lib Liang Zhang ${ }^{b}$ Ru-Xiang Xua \\ aDepartment of Neurosurgery, Affiliated Bayi Brain Hospital, PLA. Army General Hospital, Southern \\ Medical University, Beijing, 'bThe First Department of Neurosurgery, Guangdong 999 Brain Hospital, \\ Guangzhou, P.R. China
}

\section{Key Words}

Verbascoside - Signal transducer and activator of transcription $3 \cdot$ Src homology 2 domaincontaining protein tyrosine phosphatase 1 - Proliferation $\bullet$ Migration $\cdot$ Invasion $\cdot$ Apoptosis $•$ Glioblastoma

\begin{abstract}
Background/Aims: As a natural antioxidant, verbascoside (VB) is proved to be a promising method for the treatment of oxidative-stress-related neurodegenerative diseases. Thus, this study aimed to investigate the effects of VB on glioblastoma cell proliferation, apoptosis, migration, and invasion as well as the mechanism involving signal transducer and activator of transcription 3 (STAT3) and Src homology 2 domain-containing protein tyrosine phosphatase 1 (SHP-1). Methods: U87 cells were assigned to different treatments. The MTT assay was used to test cell proliferation, flow cytometry was used to detect cell apoptosis, and a Transwell assay was used for cell migration and invasion. We analyzed the glioblastoma tumor growth in a xenograft mouse model. Western blot analysis was employed to determine the protein expression of related genes. Results: Glioblastoma cells exhibited decreased cell proliferation, migration, invasion, and increased apoptosis when treated with VB or TMZ. Western blot analysis revealed elevated SHP-1 expression and reduced phosphorylated (p)-STAT3 expression in glioblastoma cells treated with VB compared with controls. Correspondingly, in a xenograft mouse model treated with VB, glioblastoma tumor volume and growth were decreased. Glioblastoma xenograft tumors treated with VB showed elevated SHP-1, Bax, cleaved W.-Q. Jia and Z.-T. Wang contributed equally to this work.
\end{abstract}

Ru-Xiang Xu and Liang Zhang 
Jia et al.: Effect of VB in Glioblastoma Via SHP-1 and STAT3

caspase-3, and cleaved PARP expression and reduced p-STAT3, Bcl-2, survivin, MMP-2, and MMP-9 expression. siRNA-SHP-1 inhibited the VB effects on glioblastoma. Conclusion: This study demonstrates that VB inhibits glioblastoma cell proliferation, migration, and invasion while promoting apoptosis via SHP-1 activation and inhibition of STAT3 phosphorylation.

(C) 2018 The Author(s)

Published by S. Karger AG, Basel

\section{Introduction}

Glioblastoma, arising from precursor or glial cells, is one of the most prevalent malignancies in gliomas and accounts for $45.6 \%$ of primary malignant brain tumors [1]. It occurs in approximately $16.1 \%$ of the Chinese population [2]. Conventional treatments including temozolomide (TMZ) and radiotherapy as well as intensive treatment (maximal resection of tumor) are usually utilized in clinical practice, and the mean overall survival time is approximately 15 months to 17 months $[3,4]$. Glioblastoma as a malignant tumor occurring in the brain usually leads to a poor prognosis, which can be attributed to glioblastoma stem cell resistance to radiochemotherapy. No matter what treatments are adopted, death occurs due to rapid proliferation of the tumor cells $[5,6]$. A previous study revealed that glioma cells exposed to chemotherapeutic agents are able to cause a more infiltrative phenotype and enhanced chemoresistance [7]. In addition, TMZ administration adjuvant to radiotherapy might promote the elimination of glioblastoma stem cells and consequently prolong the survival of patients and has been used as first-line treatment for glioblastoma patients [8, 9]. Verbascoside (VB) is a cancer therapeutic for colorectal cancer, glioma, and breast cancer [10]. Herein, TMZ was enrolled in the present study as a comparator with the function of VB.

VB was obtained from Buddleia davidii meristematic cells and was first explored to evaluate the behavior of the active ingredient in solution or in finished preparations, in light of its potential topical use, particularly in skin protection [11]. VB is a component of Cistanche (a medical plant in traditional Chinese medical science) in the form of polyphenols, and it has high antioxidant power, which is inhibitory to cell proliferation and facilitated cell apoptosis in treating colorectal cancer via the homeodomain interacting protein kinase 2 -p53 signaling pathway $[10,12]$. Inactivation of tumor suppressor phosphatases is known as a critical event in the pathogenesis of brain tumor glioblastoma [13]. Src homology 2 domain-containing protein tyrosine phosphatase 1 (SHP-1), identified as a cytosolic phosphatase, is predominantly expressed in hematopoietic and epithelial cells, serving as a negative regulator of different signaling pathways, including signal transducer and activator of transcription 3 (STAT3) [12]. STAT3 is a member of the transcription factor family, which acts to change the expression of certain genes by transducing various cytokine signals [14]. Deregulation of STAT3 promotes proliferation and invasiveness of human glioblastoma cells [13]. In particular, highly expressed phosphorylated (p)-STAT3 in cancer cell plays an essential role in the progression of cancer via downregulation by SHP-1 [15]. Therefore, in the current study, we tried to explore the underlying molecular mechanism of VB in glioblastoma involving SHP-1 and STAT3.

\section{Materials and Methods}

Ethical statement

The study was approved by the Ethics Committee of Affiliated Bayi Brain Hospital, PLA Army General Hospital, Southern Medical University.

\section{Cell culture}

Human glioblastoma cell lines U87, A172, T98G, U373, SHG-44 and U251 purchased from the Cell Center at the Basic Medicine Institute of Union Medical College (Beijing, China) were incubated with highglucose Dulbecco's modified Eagle's medium (DMEM) supplemented with 10\% fetal bovine serum (FBS) at $37^{\circ} \mathrm{C}$ under saturated humidity with $5 \% \mathrm{CO}_{2}$. Cells in the logarithmic growth phase were digested by 
trypsin before centrifugation. After the supernatant was removed, the cells were resuspended to prepare a cell suspension $\left(5.0 \times 10^{4}\right.$ cells $\left./ \mathrm{mL}\right)$. After a 24 -h incubation, $198 \mu \mathrm{L}$ of culture medium was used to replace the original medium and was added into the cell suspension. After VB was dissolved in DMSO, the gradient concentration of the VB mother liquor (clear solution without precipitation) was obtained after dilution by culture medium. In addition, $2 \mu \mathrm{L}$ of VB mother liquor at different concentrations $(0,10,20,40,60,80$, or $100 \mu \mathrm{mol} / \mathrm{L}$ ) was added into each well. A blank group (the group with only culture medium and no cells) and a negative control group, named the DMSO group (cells treated by $2 \mu \mathrm{L}$ DMSO), were set. The experiment in each group for each concentration was repeated three times. The DMSO group contained 1\% v/v DMSO.

\section{Cell grouping}

U87 and U251 cells in the logarithmic growth phase were seeded into a 24-well plate $(5 \times$ $10^{4}$ cells $/ \mathrm{mL}$ ) after digestion with trypsin. When the cell confluence reached $90 \%$, transfection was carried out. Serum-free Opti-MEM was used to dilute small interfering RNA-SHP-1 (si-SHP-1, 5'-TCCCGACAACACAATACCAGATAAATTCAAGAGATTTATCTGGTATTGTGTTGTCTTT-3') and Lipofectamine 2000. The siRNA sequence was designed by Shanghai GenePharma Co., Ltd. (Shanghai, China). Chemical modification was performed at the 3'-end and FAM fluorescence labeling at the 5 '-end. The mixture of si-SHP-1 and Lipofectamine 2000 was added into wells. After transfection for $24 \mathrm{~h}$, U87 and U251 cells were observed and photographed using an inverted fluorescence microscope (Olympus, Tokyo, Japan). A U87 cell line with relatively high transfection efficiency was used for the following experiments. Cells were treated with 50 $\mu \mathrm{mol} / \mathrm{L}$ of VB after 24-h transfection. Then, cells were grouped into a control, VB, si-SHP-1 + VB, scrambled siRNA + VB, temozolomide (TMZ) (cells treated with $300 \mu \mathrm{mol} / \mathrm{L} \mathrm{TMZ),} \mathrm{and} \mathrm{TMZ} \mathrm{+} \mathrm{VB} \mathrm{groups.} \mathrm{The} \mathrm{sequence}$ of scrambled siRNA was 5'-TCCCTTCTCCGAACGTGTCACGTTTCAGAGAACGTGACACGTTCGGAGAATT-3'.

\section{MTT assay}

After transfection for $24 \mathrm{~h}$, MTT solution $(100 \mu \mathrm{L}, 0.5 \mathrm{mg} / \mathrm{mL})$ was added into each well for a 4-h incubation. The culture medium was removed by a fluid transfer gun. Each well was supplemented with $150 \mu \mathrm{L}$ DMSO and shaken on a shaking table. Thirty minutes later, the optical density (OD) at $490 \mathrm{~nm}$ was measured using a microplate reader (Bio-Rad, Hercules, CA, USA). Cell survival rate $=\left(O D_{\text {experiment }}-O_{\text {blank }}\right)$ $/\left(\mathrm{OD}_{\text {negative control }}-\mathrm{OD}_{\text {blank }}\right) \times 100 \%$.

\section{Flow cytometry}

Cells in the logarithmic growth phase were adjusted to $1 \times 10^{6}$ cells $/ \mathrm{mL}$. The cell suspension $(0.5 \mathrm{~mL})$ was placed in a centrifuge tube, stained with $1.25 \mu \mathrm{L}$ AnnexinV-FITC (KeyGen Biotech Co., Ltd., Nanjing, Jiangsu, China) at room temperature for $15 \mathrm{~min}$ avoiding light, and centrifuged for $5 \mathrm{~min}$ at $1000 \mathrm{rpm}$, followed by the supernatant being discarded. Cells were resuspended with $0.5 \mathrm{~mL}$ cold binding buffer. Subsequently, $10 \mu \mathrm{L}$ propidium iodide (PI) was added. Flow cytometry (BD, Franklin Lakes, NJ, USA) was used to detect the cell apoptosis. Viable cells (Annexin $\mathrm{V}^{-} / \mathrm{PI}^{-}$) are located in the lower left quadrant of each panel; early apoptotic cells (Annexin $\mathrm{V}^{+} / \mathrm{PI}^{-}$), in the lower right quadrant; and late apoptotic cells (Annexin $\mathrm{V}^{+} / \mathrm{PI}^{+}$), in the upper right quadrant. The cell apoptosis rate was considered the sum of the cell percentage in the lower right and upper right quadrants.

\section{Transwell assay}

Tumor cells were placed in the apical chamber, and the FBS chemokine was placed in the basolateral chamber. Tumor cells migrated to the apical chamber with a high nutrient content, and the number of cells entering the basolateral chamber reflected the migration ability of the tumor cells. The cell density was adjusted to $1 \times 10^{5}$ cells $/ 100 \mu \mathrm{L}$ by serum-free culture media. The cell suspension $(100 \mu \mathrm{L})$ was seeded in the apical chamber of 24-well Transwell chambers (BD, Franklin Lakes, NJ, USA). The basolateral chamber was supplemented with $500 \mu \mathrm{L}$ of culture medium containing $10 \%$ FBS. After incubation under saturated humidity with $5 \% \mathrm{CO}_{2}$ at $37^{\circ} \mathrm{C}$ for $24 \mathrm{~h}$, cells in the apical chamber were removed using cotton swabs. The chambers were immersed in $4 \%$ paraformaldehyde for $15 \mathrm{~min}$, washed with phosphate-buffered saline (PBS), and stained with crystal violet for $30 \mathrm{~min}$. Next, the chambers were washed in purified water and replaced in the 24 -well plate. Crystal violet was dissolved in $33 \%$ acetic acid, followed by a $100 \mu \mathrm{L}$ solution being soaked into a 96-well plate. The OD at $570 \mathrm{~nm}$ was measured using a microplate reader as the number of migrating cells. Matrigel glue mimicked the extracellular matrix. Tumor cells needed to digest this layer of 
matrix glue to pass through the membrane and enter the Transwell chamber. The number of cells entering the basolateral chamber reflected the invasive ability of tumor cells. Matrigel $(40 \mu \mathrm{L})$ was dissolved at $4^{\circ} \mathrm{C}$ and then added into cold Transwell chambers for a 1-h incubation. Following the procedures for detecting migration, the OD at $570 \mathrm{~nm}$ was used to determine the number of invasive cells.

Xenograft tumors in nude mice

A total of 25 female BALB/c nude mice were purchased from Shenzhen Huafukang Bioscience Co., Inc., (Shenzhen, China). A xenograft tumor was generated using a cell-inoculated suspension. U87 cells in the logarithmic growth phase were resuspended to $2.5 \times 10^{5}$ cells/L with PBS after digestion by trypsin. The cell suspension $(100 \mu \mathrm{L})$ was subcutaneously injected into the right back of five nude mice using a $100-\mu \mathrm{L}$ microinjector. Massive inoculation of a piece of solid tumor tissue was performed. Nude mice with tumors were selected to obtain tumor tissue after anesthesia under sterile conditions, followed by the fatty and necrotic tissues being removed. Tumor tissue was cut into $1 \mathrm{~mm}^{3}$. Nude mice were anesthetized and sterilized, an incision was made on the right back, and the tissue block was inoculated using ophthalmic tweezers. The skin was sutured and sterilized. Ten days after inoculation, nude mice were assigned to a model group (daily intraperitoneal injection of PBS), VB group (daily intraperitoneal injection of $80 \mathrm{mg} /$ $\mathrm{kg} \mathrm{VB}$ ), si-SHP-1 + VB group (daily intraperitoneal injection of $200 \mu \mathrm{L}$ si-SHP-1 and $80 \mathrm{mg} / \mathrm{kg} \mathrm{VB}$ ), and scrambled siRNA + VB group (daily intraperitoneal injection of $200 \mu \mathrm{L}$ scrambled siRNA and $80 \mathrm{mg} / \mathrm{kg} \mathrm{VB}$ ), with 5 mice in each group. Injections were performed continuously for 20 days. Mouse weight and tumor volume were calculated every 4 days. The maximum diameter (a) and minimum diameter (b) of tumors in the mice were measured using a Vernier caliper. Tumor volume was calculated using the formula: $\mathrm{V}\left(\mathrm{mm}^{3}\right)=$ $1 / 2 \mathrm{ab}^{2}$. The mice were sacrificed, and the tumor tissue was collected for western blot analysis.

\section{Western blot analysis}

Total protein was extracted from xenograft tumors using RIPA lysate buffer (Beyotime Biotechnology Co., Ltd., Shanghai, China). The Bradford method (Thermo Fisher Scientific, Waltham, MA, USA) was adopted for quantitative analysis of protein. Then, $50 \mu$ g of protein was separated by $12 \%$ sodium dodecyl sulfatepolyacrylamide gel electrophoresis (SDS-PAGE) and transferred onto polyvinylidene fluoride (PVDF) membranes. The membranes were blocked with $5 \%$ skim milk, shaken for $1 \mathrm{~h}$ at $37^{\circ} \mathrm{C}$ on a shaking table and then incubated overnight at $4^{\circ} \mathrm{C}$ with SHP-1, STAT3, p-STAT3, cleaved caspase-3, cleaved PARP, Bax, Bcl2, survivin, MMP-2, MMP-9, or GAPDH rabbit anti-mouse monoclonal antibody (Cell Signaling Technology, Beverly, MA, USA) at 1: 1000 dilution. After washing with PBS Tween 20 (PBST) three times (5 min each), the membranes were incubated with horseradish peroxidase (HRP)-conjugated goat anti-rabbit antibody (1: 4000, Cell Signaling Technology, USA) for $2 \mathrm{~h}$, and then washed with PBST three times (5 min each). Proteins were detected by using luminol reagent and peroxide solution (Millipore, Billerica, MA, USA) at a ratio of 1: 1. Images were acquired for further analysis. The average absorbance ratio of target protein bands to the internal reference (GAPDH) band was considered the relative protein expression. These procedures were also applied to the cell experiment. The experiment was repeated three times.

\section{Statistical analysis}

Statistical analysis was conducted using SPSS 21.0 (IBM Corp., Armonk, NY, USA). Data are presented as the mean \pm standard deviation. Comparisons between two groups were analyzed using $t$-tests. Comparisons among multiple groups were assessed using one-way analysis of variance. Numerical data are expressed as a percentage or rate and were analyzed by chi-square tests. A $p<0.05$ was considered statistically significant.

\section{Results}

\section{VB inhibited U87 and U251 cell proliferation}

U87, A172, T98G, U373, SHG-44, and U251 cell lines were treated with VB in a gradient of concentrations and time points. As shown in Fig. 1, VB inhibited U87, A172, T98G, U373, SHG-44, and U251 cell proliferation depending on the time and concentration. In the subsequent experiment, we selected the U87 and U251 cells. When U87 and U251 cell lines were treated with VB for $48 \mathrm{~h}$ and the drug concentration was between $40 \mu \mathrm{mol} / \mathrm{L}$ and 60 
Fig. 1. Survival rate in U87, A172, T98G, U373, SHG-44, and U251 cell lines following VB treatment. When $U 87$ and $U 251$ cell lines were treated with VB for $48 \mathrm{~h}$ and the drug concentration was between $40 \mu \mathrm{mol} / \mathrm{L}$ and $60 \mu \mathrm{mol} / \mathrm{L}$, the survival rate decreased the most. Note: Panel A-F: The survival rate of different concentrations of VB $(0,10,10,20,40,60,80,100 \mu \mathrm{mol}$ /L) on U87, A172, T98G, U373, SHG-44, and U251 cells at different times, respectively. Cell survival

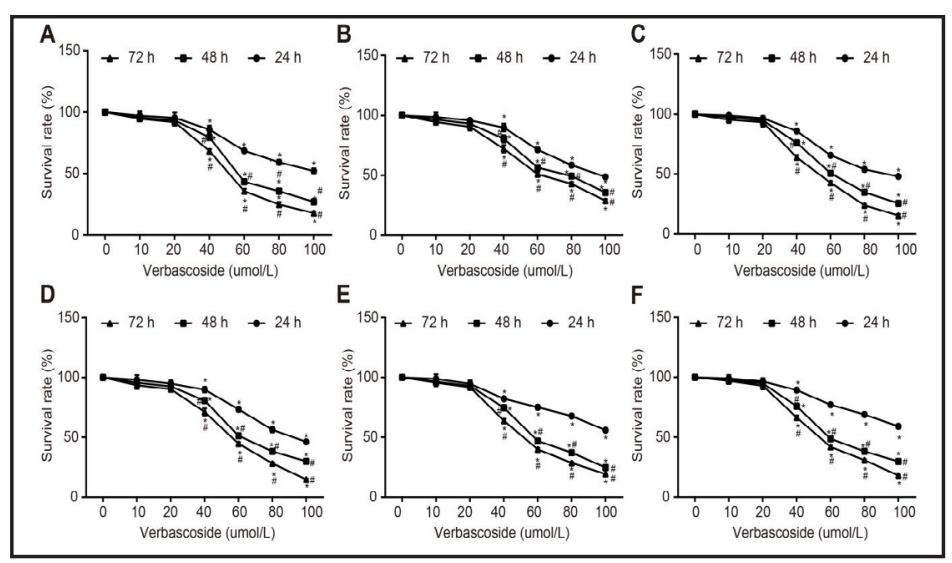
rate $=\left(O D_{\text {experiment }}-O D_{\text {blank }}\right) /(O D$ - OD ${ }_{\text {blank }}$ ) $\times 100 \%$. The experimental group was treated with different drug concentrations, the blank control group was a cell-free pure medium group, and the negative control group was a solvent group. Data are presented as the mean \pm standard deviation and were assessed using one-way analysis of variance. $\mathrm{N}=3$. $^{*}, \mathrm{p}<0.05$ compared with the former VB concentration at the same time point; \#, p<0.05 compared with the former time point with the same VB concentration.

Fig. 2. Assessment of the proliferation of U87 cells among six groups. VB promoted the anti-proliferation effect of TMZ in U87 cells. Note: Panel A, images showing the transfection efficiency of the U87 and U251 cell lines; $2 \mu \mathrm{L}$ Invitrogen
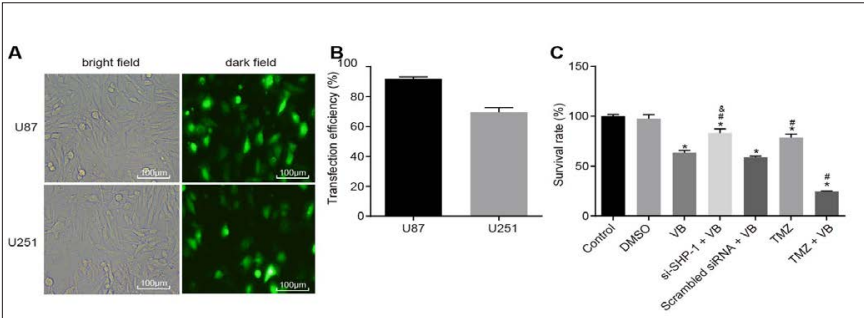
Lipofectamine $2000+3 \mu \mathrm{L}$ FAM-

siRNA (concentration, $20 \mathrm{pm} / \mu \mathrm{L}$ ) was added to the cells; after $24 \mathrm{~h}$, the state of the cells and the number of fluorescent cells were observed in the field of light and in the field of fluorescence respectively; green fluorescence is a fluorescent siRNA successfully transferred into a cell. Panel B, U87 and U251 transfection efficiency. Panel C: comparing cell survival in six groups; data are presented as the mean \pm standard deviation and were assessed using one-way analysis of variance. $\mathrm{N}=3 .{ }^{*}, \mathrm{p}<0.05$ compared with the control group; \#, $\mathrm{p}<0.05$ compared with the VB group; \&, p $<0.05$ compared with the scramble siRNA + VB group.

$\mu \mathrm{mol} / \mathrm{L}$, the survival rate decreased the most $(p<0.05)$. Hence, the concentration was set 50 $\mu \mathrm{mol} / \mathrm{L}$ for the following experiments, and the function time was set $48 \mathrm{~h}$.

\section{A U87 cell line with relatively high transfection efficiency was selected and its proliferation was inhibited after transfection}

Glioma cell lines U251 and U87 were transfected in accordance with the experimental method. All siRNAs were labeled with FAM fluorescence. After transfection for $24 \mathrm{~h}$, the transfection efficiency of the cells was observed under a fluorescence inverted microscope and was also tested by RT-qPCR. The results revealed that the transfection efficiency of glioma cell line U87 (91.83\%) was superior to that of glioma cell line U251 (69.51\%), and the mRNA expression of SHP-1 in U87 cells transfected with si-SHP-1 was significantly lower than that in U251 cells. Thus, the U87 cell line was selected (Fig. 2A and B). After the U87 cells were transfected with SHP-1 siRNA and scrambled siRNA for $24 \mathrm{~h}$, the cells were treated with $50 \mu \mathrm{mol} / \mathrm{L} \mathrm{VB}$, and then MTT was used to detect the OD490. Cell survival rate $=$ (OD experiment $\left.-O D_{\text {blank }}\right) /\left(O D_{\text {negative control }}-O D_{\text {blank }}\right) \times 100 \%$. As shown in Fig. 2C, in comparison to the control group, no significant difference was found for the cell survival rate in the DMSO group, while the cell survival rate was much lower in the VB and TMZ groups $(p<0.001)$.

\section{KARGER}


The cell survival rate in the si-SHP-1 + VB group was increased compared with that in the VB group $(p<0.001)$. The cell survival rate was higher in the si-SHP-1 + VB group than in the scrambled siRNA + VB groups $(p<0.001)$. These results showed that si-SHP-1 suppressed the inhibitory effect of VB on U87 cell proliferation. The cell survival rate in the TMZ group was elevated compared with that in the VB group (all $p=0.0002$ ). The cell survival rate in the TMZ + VB group was lower than that in the TMZ and VB groups $(p<0.0001)$, indicating that VB potentiated the anti-proliferation effect of TMZ on U87 cells.

\section{VB induced U87 cell apoptosis}

Flow cytometry was adopted to assess the effects of VB on cell apoptosis. As shown in Fig. 3, the cell apoptosis rate was increased in the VB and TMZ groups compared with that in the control group $(p<0.05)$. Compared with that in the VB group, the apoptosis rate was lower in the si-SHP-1 + VB group ( $p<0.05)$, while no significant difference was found in the scrambled siRNA + VB group $(p>0.05)$. Compared with that in the scrambled siRNA + VB group, a decreased apoptosis rate was observed in the si-SHP-1 + VB group $(p<0.05)$. The data suggested that si-SHP-1 inhibited VB-induced U87 cell apoptosis. The apoptosis rate in the TMZ group was decreased compared with that in the VB group and increased compared with that in the control group $(p<0.05)$. A higher apoptosis rate was detected in the TMZ + VB group than in the TMZ and VB groups $(p<0.05)$. These results indicate that VB potentiated TMZ-induced U87 cell apoptosis.

\section{VB inhibited U87 cell migration and invasion}

A Transwell assay was applied to evaluate the effects of $\mathrm{VB}$ on cell migration and invasion (Fig. 4). Cell invasion and migration decreased in the VB and TMZ groups $(p<0.05)$ compared with the levels in the control group. Cell invasion and migration in the si-SHP-1 + VB group increased compared with the levels in the VB group $(p<0.05)$. No differences in cell invasion and migration were observed between the VB and scrambled siRNA + VB groups $(p>0.05)$. Compared with the levels in the scrambled siRNA + VB group, increased cell migration and invasion were observed in the si-SHP-1 + VB group $(p<0.05)$. These results suggested that si-SHP-1 partly suppressed the inhibition of VB on the invasion and migration of U87 cells. The number of migratory and invasive cells in the TMZ group decreased compared with those in the control group but increased compared with those in the VB group $(p<0.05)$. Elevated migration and invasion were found in the TMZ and VB groups compared with the levels in the TMZ + VB group $(p<0.05)$. The results demonstrated that VB potentiated the inhibition of TMZ on U87 cell migration and invasion.

Fig. 3. Flow cytometry determination revealed the induced U87 cell apoptosis after VB treatment. Note: Panel A, U87 cell apoptosis detected by flow cytometry; Panel B, histogram of the cell apoptosis rate in six groups. Data are presented as the mean \pm standard deviation and were assessed using one-way analysis of variance. $\mathrm{N}=3 .{ }^{*}, \mathrm{p}<0.05$ compared with the control group; \#, $\mathrm{p}<0.05$

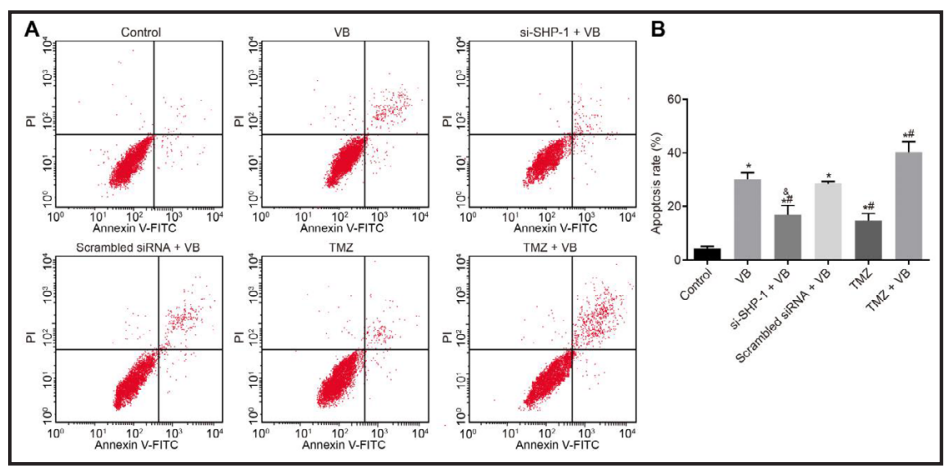
compared with the VB group; \&, p $<0.05$ compared with the scramble siRNA + VB group. 
Fig. 4. U87 cell migration and invasion were inhibited after VB treatment. Note: Panel A, images showing the migration of glioblastoma U87 cells detected by a Transwell assay; Panel $\mathrm{B}$, histogram comparing the migration of glioblastoma U87 cells among the six groups; Panel $\mathrm{C}$, images showing the invasion of glioblastoma U87 cells detected by the Transwell assay; Panel D, histogram comparing the invasion of glioblastoma U87 cells among the six groups. Data are presented as the mean \pm standard deviation and were assessed using one-
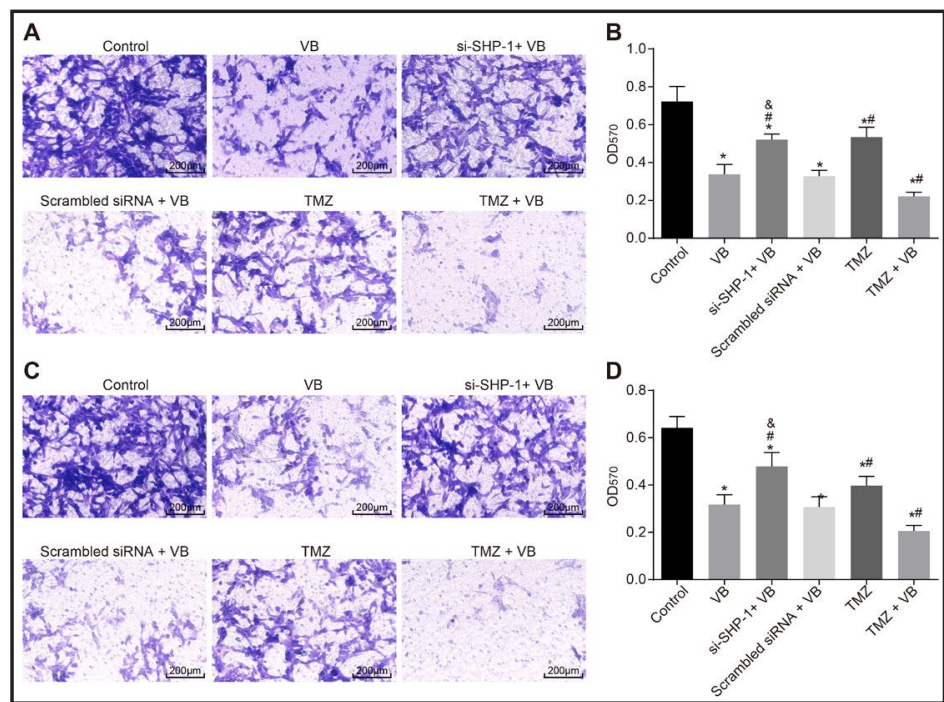

$\mathrm{p}<0.05$ compared with the control group; \#, $\mathrm{p}<0.05$ compared with the VB group; \&, $\mathrm{p}<0.05$ compared with the scramble siRNA + VB group.

Fig. 5. Protein expression of SHP1, p-STAT3, and STAT3 in U87 cells after transfection. Note: Panel A, protein bands observed after western blot analysis; Panel B, histogram showing the difference of protein expression of SHP-1, p-STAT3, and STAT3 in the six groups; Data are presented as the mean \pm standard deviation

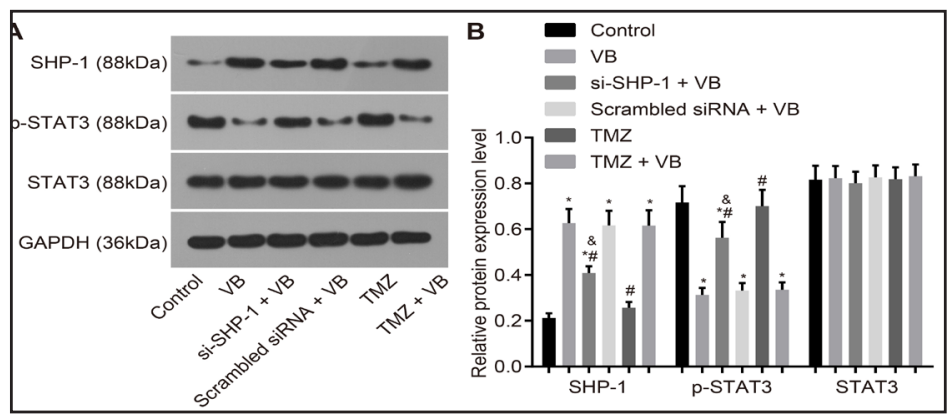
and were assessed using one-way analysis of variance. $\mathrm{N}=3 .{ }^{*}, \mathrm{p}<0.05$ compared with the control group; \#, $\mathrm{p}<0.05$ compared with the VB group; \&, p<0.05 compared with the scramble siRNA + VB group.

Fig. 6. In vivo experiment demonstrating suppressed mouse weight and xenograft tumor volume following VB treatment. Note: Panel A, body weight of nude mice; Panel B, tumor volume; Data are presented as the mean \pm standard deviation and were assessed using one-way analysis of
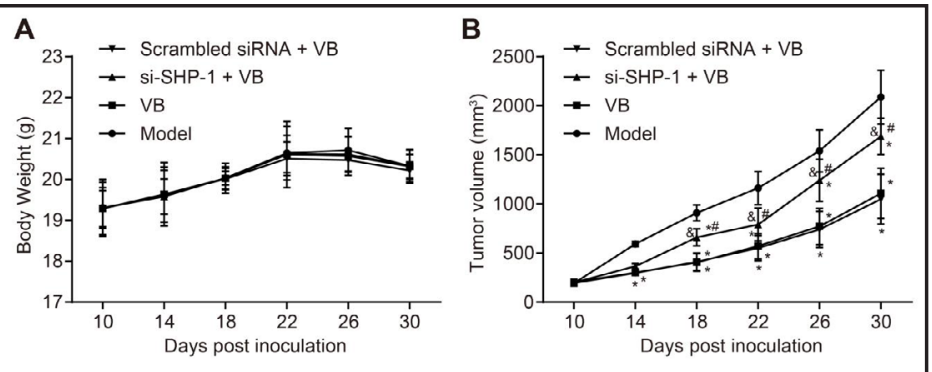
variance. $\mathrm{N}=3 .^{*}, \mathrm{p}<0.05$ compared with the control group; \#, p $<0.05$ compared with the VB group; \&, p $<0.05$ compared with the scramble siRNA + VB group.

Protein expression of SHP-1, p-STAT3 and STAT3 in U87 cells following transfection

Higher SHP-1 expression and lower p-STAT3 expression were detected in the VB, siSHP-1 + VB, scrambled siRNA + VB and TMZ + VB groups than in the control group $(p<$ 0.05); there was no difference found between the control group and the TMZ group ( $p$ $>0.05)$. In comparison to the levels in the VB group, SHP-1 expression was reduced and 
Jia et al.: Effect of VB in Glioblastoma Via SHP-1 and STAT3

p-STAT3 expression was elevated in the si-SHP-1 + VB group $(p<0.05)$. No difference was observed in STAT3 expression among the six groups $(p>0.05)$. Compared with the levels in the scrambled siRNA + VB group, increased level of SHP-1 and reduced level of p-STAT3 were observed in the si-SHP-1 + VB group $(p<0.05)$ (Fig. 5). The results showed that transfection of shp-1 siRNA could partially reverse the increase in SHP-1 expression and the decrease in p-STAT3 expression induced by VB.

\section{Xenograft tumor growth in nude mice}

The body weight and tumor volume of each group of nude mice were observed. No difference was found in mouse weight among the six groups (Fig. 6A) $(p>0.05)$. As shown in Fig. 6B, compared with that in the model group, the volume of the xenograft tumor was decreased in the VB, si-SHP-1 + VB, and scrambled siRNA + VB groups $(p<0.05)$. In comparison to that in the VB group, the volume of xenograft tumors was elevated in the si-SHP-1 + VB group $(p<0.05)$. There was no difference between the VB and scrambled siRNA + VB groups $(p>0.05)$. Compared with that in the scrambled siRNA + VB group, an increased volume of xenograft tumors was observed in the si-SHP-1 + VB group $(p<0.05)$. These findings provided evidence that VB inhibited xenograft tumor growth.

\section{Expression of SHP-1 and STAT3 signaling pathway-related proteins in xenograft tumor} tissue among four groups

The nude mice were sacrificed after the tumor volume was measured 30 days after tumor transplantation, and protein was extracted to detect the expression of related proteins by western blot analysis. Compared with the model group, the VB group had higher SHP-1 expression and lower p-STAT3 expression. Expression of the pro-apoptosis-related protein (Bax, cleaved caspase-3, and cleaved PARP) STAT3 downstream was increased in the VB group, but anti-apoptosis protein (Bcl-2), and cell survival-related protein (survivin) as well as tumor cell migration-related proteins (MMP-2 and MMP-9) were decreased ( $p>$ 0.05). Compared with the levels in the scrambled siRNA + VB group, decreased expression levels of SHP-1, Bax, cleaved caspase- 3 , and cleaved-PAPRP but increased expression levels of p-STAT3, Bcl-2, survivin, MMP-2, and MMP-9 were observed in the si-SHP-1 + VB group $(p<0.05)$. si-SHP-1 treatment partially reversed the regulation of VB on related proteins. These results indicate that VB inhibits STAT3 phosphorylation by upregulating SHP-1 expression and thus altering STAT3 downstream signaling to influence the biological activity of glioblastoma (Fig. 7).

Fig. 7. Western blot analysis showing that VB inhibited STAT3 phosphorylation by upregulating the expression of SHP1 and further regulated the downstream target proteins of STAT3. Note: Panel A, protein bands observed following western blot analysis; Panel B, histogram showing the relative expression of related proteins among four groups; Data are presented as the mean \pm standard deviation and were assessed using one-way analysis of variance. $\mathrm{N}=3$. *, $\mathrm{p}<0.05$ compared with the model group; \#, $\mathrm{p}<0.05$ compared with the VB group; \&, $p<0.05$ compared with the scramble siRNA + VB group.

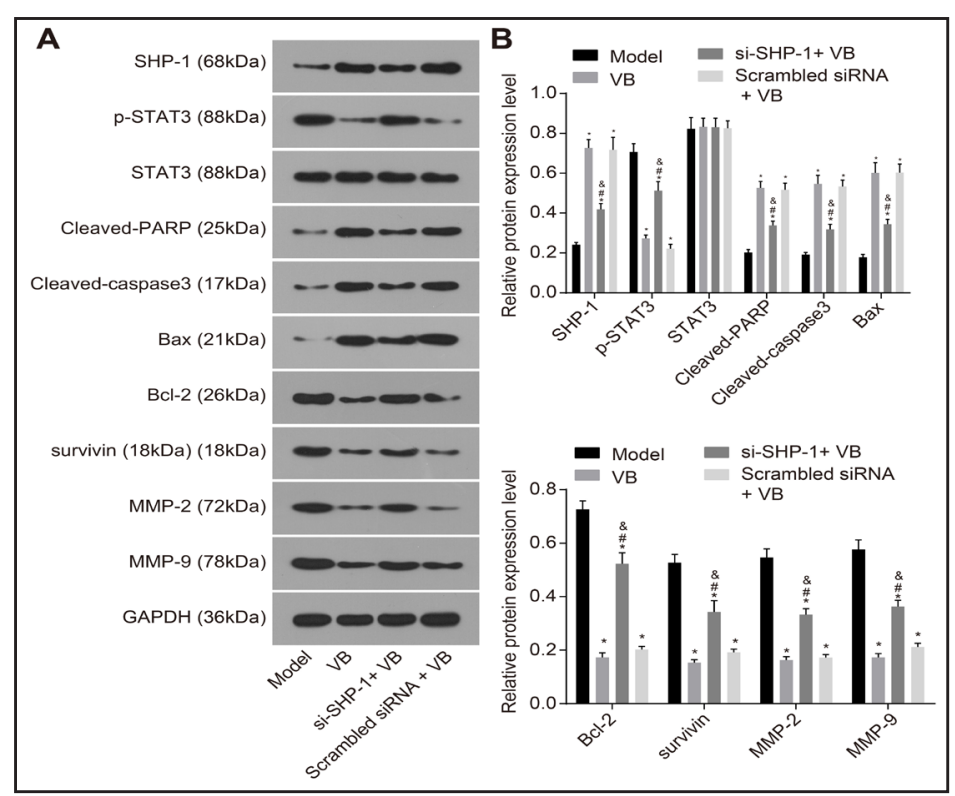




\section{Discussion}

Glioblastoma is a highly aggressive brain tumor with a poor prognosis [16]. VB, also known as acteoside, has been described in the literature for its function as a regulator of various biological activities, such as tumor-suppression, antioxidative and antimicrobial activities [17]. However, there is not yet enough information available to predict its role in glioblastoma. This study aimed to investigate the effect of VB on cell proliferation, migration, invasion, and apoptosis in glioblastoma.

We observed increased SHP-1 expression and inhibition of STAT3 phosphorylation in glioblastoma following VB treatment. SHP-1 is a cytoplasmic tyrosine phosphatase. A homozygous mutation of the SHP-1 gene, which leads to complete loss of SHP-1 expression, causes severe dysregulation of leukocyte development and systemic autoimmunity. Loss of SHP-1 has been observed in a proportion of T-cell lymphomas, including the majority of the ALK ${ }^{+}$ALCL cases, as an indicator of its role in tumor suppression [18]. Moreover, p-STAT may form homo- or heterodimers and transpose into the nucleus, thus stimulating gene transcription. STAT3, as one of the STAT proteins, can be activated by the IL-6 cytokine family, which phosphorylates STAT3 at Tyr705 [19]. Constitutive and inducible activation of STAT3 signaling promotes carcinogenesis in many human cancers such as acute myeloid leukemia. Negative regulators, such as protein tyrosine phosphatase SHP-1, may suppress the activated STAT3 signaling [20]. Additionally, 3 phosphatases, TC45, SHP-1 and SHP-2, are the primary contributory enzymes of UVB-mediated STAT3 dephosphorylation, and this may be a protective mechanism against UV skin carcinogenesis [21]. Moreover, a previous study also revealed that the loss of SHP- 1 could lead to the pathogenesis of ALK+ anaplastic largecell lymphoma through enhancement of tyrosine phosphorylation and activation of JAK3/ STAT3 [22]. In line with our study, Han et al. demonstrated that higher levels of SHP1 seen subsequently correlate with substantial decreases in pSTAT3 [23]. Therefore, we inferred a negative relationship between SHP-1 and STAT3 phosphorylation and that these proteins may be involved in glioblastoma.

Following transfection and VB treatment, expression of Bax, cleaved caspase-3, and cleaved PARP were increased in glioblastoma tumor tissues. Expression of Bcl-2, survivin, MMP-2, and MMP-9 were decreased. As cytosolic proteins, Bcl-2 targets the nucleus and inhibits apoptosis with a lipid-anchoring domain, while Bax, one of the members of the Bcl-family, homodimerizes and forms heterodimers with Bcl-2 protein to reduce the antiapoptotic effect of Bcl-2, leading to apoptotic death [24]. As an apoptosis effector enzyme, Caspase-3 is triggered by intrinsic (mitochondria) and extrinsic pathways. Studies show that docosahexaenoic acid activates Caspase- 3 and decreases another apoptosis marker, PARP. In the intrinsic apoptosis pathway, Bax translocates to the cell membrane and complexes with Bcl-2, thereby protecting the mitochondrial membrane, which in turn results in release of cytochrome c, ultimately leading to apoptosis [25]. MMP-2 and MMP-9 through leukemic cells may enhance the penetrability of the blood-brain barrier via disrupting endothelial tight junction proteins in the central nervous system (CNS), thereby resulting in invasion of leukemic cells to the CNS in acute leukemia [26]. Sulforaphane, which has antioxidant and antitumor activities and viability inhibition effects, promotes apoptosis of U251 MG cells by increasing annexin V-binding capacity, Bad, Bax, cytochrome C expression and by reducing Bcl-2 and survivin expression, as well as inhibiting invasion in U251 MG cells via upregulating E-cadherin and downregulating MMP-2, MMP-9, and Galectin-3 [27].

Reduced cell proliferation, percentage of cells in S phase, migration, invasion, and tumor growth/volume are observed following VB administration, but an enhanced percentage of cells in G0/G1 and an increase in apoptosis are also observed. As interferon signaling molecules, JAKs and STATs also signal in response to other cytokines and growth factors, thus regulating various cellular functions such as cell proliferation, apoptosis, and inflammation. The transient activation of the JAK-STAT signaling pathway in ganglioside-stimulated microglia is due to phosphorylation and lipid raft-regulated SHP-2 associated with JAK-2, indicating a regulatory mechanism of SHP-2 in activated microglia [28]. Interestingly, SHP- 
2 silencing has been observed to induce increased $\beta$-galactosidase staining, significant morphological changes, and a reduced number of cells in the G2/M and S phases, indicating a remarkable inhibitory effect of SHP-2 silencing because of enhanced senescence, not necrosis or apoptosis. SHP-2 may in part advance the growth of glioblastoma cells via inhibiting cellular senescence [29]. In addition, 1'-acetoxychavicol acetate (ACA) may suppress both constitutive and interleukin-6-inducible STAT3 activation, decrease the accumulation of STAT3 in the nucleus and its DNA-binding activity, and upregulate SHP-1, thus inhibiting cancer cell migration and invasion [30]. Therefore, we can infer that SHP-1 and STAT3 may participate in cell proliferation, migration, invasion, and apoptosis in glioblastoma.

Generally, the present study suggests that VB exerts effects on the cell viability of glioblastoma through SHP-1 upregulation to inhibit phosphorylation of STAT3 and to mediate relevant proteins downstream of STAT3. As a conclusion drawn by a previous study, SHP-1 inhibits phosphorylation of STAT3 (a member of the STAT family and a regulator of transcription), to inactivate STAT3 [31]. Furthermore, it is noticeable that concentration and exposure time are considered key factors when utilizing VB. These findings may open novel avenues for future therapies. On the other hand, the shortage of sufficient data from in vivo animal experiments is also noteworthy. In view of the time cycle and funding reasons, this paper does not involve genetically modified animals, which is also our further research direction.

\section{Acknowledgements}

We would like to express sincere appreciation to the reviewers for critical comments on this article.

\section{Disclosure Statement}

The authors declare to have no competing interests.

\section{References}

1 Ostrom QT, Gittleman H, Liao P, Rouse C, Chen Y, Dowling J, Wolinsky Y, Kruchko C, Barnholtz-Sloan J: CBTRUS statistical report: primary brain and central nervous system tumors diagnosed in the United States in 2007-2011. Neuro Oncol 2014;16 Suppl 4:iv1-63.

-2 Yan W, Zhang W, You G, Bao Z, Wang Y, Liu Y, Kang C, You Y, Wang L, Jiang T: Correlation of IDH1 mutation with clinicopathologic factors and prognosis in primary glioblastoma: a report of 118 patients from China. PLoS One 2012;7:e30339.

-3 Preusser M, Lim M, Hafler DA, Reardon DA, Sampson JH: Prospects of immune checkpoint modulators in the treatment of glioblastoma. Nat Rev Neurol 2015;11:504-514.

-4 Kijima N, Hosen N, Kagawa N, Hashimoto N, Kinoshita M, Oji Y, Sugiyama H, Yoshimine T: Wilms' tumor 1 is involved in tumorigenicity of glioblastoma by regulating cell proliferation and apoptosis. Anticancer Res 2014;34:61-67.

5 Prados MD, Byron SA, Tran NL, Phillips JJ, Molinaro AM, Ligon KL, Wen PY, Kuhn JG, Mellinghoff IK, de Groot JF, Colman H, Cloughesy TF, Chang SM, Ryken TC, Tembe WD, Kiefer JA, Berens ME, Craig DW, Carpten JD, Trent JM: Toward precision medicine in glioblastoma: the promise and the challenges. Neuro Oncol 2015;17:1051-1063.

-6 Friesen C, Hormann I, Roscher M, Fichtner I, Alt A, Hilger R, Debatin KM, Miltner E: Opioid receptor activation triggering downregulation of cAMP improves effectiveness of anti-cancer drugs in treatment of glioblastoma. Cell Cycle 2014;13:1560-1570. 


\section{Cellular Physiology Cell Physiol Biochem 2018;47:1871-1882 \begin{tabular}{l|l} 
and Biochemistry DOI: 10.1159/000491067 & $\begin{array}{l}\text { (c) 2018 The Author(s). Published by S. Karger AG, Basel } \\
\text { www.karger.com/cpb }\end{array}$
\end{tabular}

7 Auffinger B, Tobias AL, Han Y, Lee G, Guo D, Dey M, Lesniak MS, Ahmed AU: Conversion of differentiated cancer cells into cancer stem-like cells in a glioblastoma model after primary chemotherapy. Cell Death Differ 2014;21:1119-1131.

8 Beier D, Rohrl S, Pillai DR, Schwarz S, Kunz-Schughart LA, Leukel P, Proescholdt M, Brawanski A, Bogdahn U, Trampe-Kieslich A, Giebel B, Wischhusen J, Reifenberger G, Hau P, Beier CP: Temozolomide preferentially depletes cancer stem cells in glioblastoma. Cancer Res 2008;68:5706-5715.

-9 Brandes AA, Franceschi E, Tosoni A, Benevento F, Scopece L, Mazzocchi V, Bacci A, Agati R, Calbucci F, Ermani M: Temozolomide concomitant and adjuvant to radiotherapy in elderly patients with glioblastoma: correlation with MGMT promoter methylation status. Cancer 2009;115:3512-3518.

10 Zhou L, Feng Y, Jin Y, Liu X, Sui H, Chai N, Chen X, Liu N, Ji Q, Wang Y, Li Q: Verbascoside promotes apoptosis by regulating HIPK2-p53 signaling in human colorectal cancer. BMC Cancer 2014;14:747.

11 Vertuani S, Beghelli E, Scalambra E, Malisardi G, Copetti S, Dal Toso R, Baldisserotto A, Manfredini S: Activity and stability studies of verbascoside, a novel antioxidant, in dermo-cosmetic and pharmaceutical topical formulations. Molecules 2011;16:7068-7080.

-12 Pesce M, Franceschelli S, Ferrone A, De Lutiis MA, Patruno A, Grilli A, Felaco M, Speranza L: Verbascoside down-regulates some pro-inflammatory signal transduction pathways by increasing the activity of tyrosine phosphatase SHP-1 in the U937 cell line. J Cell Mol Med 2015;19:1548-1556.

13 de la Iglesia N, Konopka G, Lim KL, Nutt CL, Bromberg JF, Frank DA, Mischel PS, Louis DN, Bonni A: Deregulation of a STAT3-interleukin 8 signaling pathway promotes human glioblastoma cell proliferation and invasiveness. J Neurosci 2008;28:5870-5878.

14 Vogel TP, Milner JD, Cooper MA: The Ying and Yang of STAT3 in Human Disease. J Clin Immunol 2015;35:615-623.

15 Fan LC, Teng HW, Shiau CW, Lin H, Hung MH, Chen YL, Huang JW, Tai WT, Yu HC, Chen KF: SHP-1 is a target of regorafenib in colorectal cancer. Oncotarget 2014;5:6243-6251.

16 Liu X, Wang X, Du W, Chen L, Wang G, Cui Y, Liu Y, Dou Z, Wang H, Zhang P, Chang L, Yi L, Cai J, Jiang C: Suppressor of fused (Sufu) represses Gli1 transcription and nuclear accumulation, inhibits glioma cell proliferation, invasion and vasculogenic mimicry, improving glioma chemo-sensitivity and prognosis. Oncotarget 2014;5:11681-11694.

17 Etemad L, Zafari R, Moallem SA, Vahdati-Mashhadian N, Skouei Shirvan Z, Hosseinzadeh H: Teratogenic Effect of Verbascoside, Main Constituent of Lippia citriodora Leaves, in Mice. Iran J Pharm Res 2016;15:521-525.

18 Hegazy SA, Wang P, Anand M, Ingham RJ, Gelebart P, Lai R: The tyrosine 343 residue of nucleophosmin (NPM)-anaplastic lymphoma kinase (ALK) is important for its interaction with SHP1, a cytoplasmic tyrosine phosphatase with tumor suppressor functions. J Biol Chem 2010;285:19813-19820.

19 Suzuki S, Tanaka K, Nogawa S, Dembo T, Kosakai A, Fukuuchi Y: Phosphorylation of signal transducer and activator of transcription-3 (Stat3) after focal cerebral ischemia in rats. Exp Neurol 2001;170:63-71.

20 Bi L, Yu Z, Wu J, Yu K, Hong G, Lu Z, Gao S: Honokiol Inhibits Constitutive and Inducible STAT3 Signaling via PU.1-Induced SHP1 Expression in Acute Myeloid Leukemia Cells. Tohoku J Exp Med 2015;237:163-172.

21 Kim DJ, Tremblay ML, Digiovanni J: Protein tyrosine phosphatases, TC-PTP, SHP1, and SHP2, cooperate in rapid dephosphorylation of Stat3 in keratinocytes following UVB irradiation. PLoS One 2010;5:e10290.

-22 Han Y, Amin HM, Franko B, Frantz C, Shi X, Lai R: Loss of SHP1 enhances JAK3/STAT3 signaling and decreases proteosome degradation of JAK3 and NPM-ALK in ALK+ anaplastic large-cell lymphoma. Blood 2006;108:2796-2803.

23 Han Y, Amin HM, Frantz C, Franko B, Lee J, Lin Q, Lai R: Restoration of shp1 expression by 5-AZA-2'deoxycytidine is associated with downregulation of JAK3/STAT3 signaling in ALK-positive anaplastic large cell lymphoma. Leukemia 2006;20:1602-1609.

24 Xie Z, Koyama T, Suzuki J, Fujii Y, Togashi H, Sawa H, Nagashima K: Coronary reperfusion following ischemia: different expression of bcl-2 and bax proteins, and cardiomyocyte apoptosis. Jpn Heart J 2001;42:759-770.

25 Yin Y, Sui C, Meng F, Ma P, Jiang Y: The omega-3 polyunsaturated fatty acid docosahexaenoic acid inhibits proliferation and progression of non-small cell lung cancer cells through the reactive oxygen speciesmediated inactivation of the PI3K / Akt pathway. Lipids Health Dis 2017;16:87. 


\section{Cellular Physiology Cell Physiol Biochem 2018;47:1871-1882 \begin{tabular}{l|l} 
DOI: 10.1159/000491067 & $\begin{array}{l}\text { O 2018 The Author(s). Published by S. Karger AG, Basel } \\
\text { www.karger.com/cpb }\end{array}$ \\
\hline
\end{tabular}}

Jia et al.: Effect of VB in Glioblastoma Via SHP-1 and STAT3

26 Das T, Bhattacharya S, Biswas A, Gupta SD, Gomes A, Gomes A: Inhibition of leukemic U937 cell growth by induction of apoptosis, cell cycle arrest and suppression of VEGF, MMP-2 and MMP-9 activities by cytotoxin protein NN-32 purified from Indian spectacled cobra (Naja naja) venom. Toxicon 2013;65:1-4.

-27 Zhang Z, Li C, Shang L, Zhang Y, Zou R, Zhan Y, Bi B: Sulforaphane induces apoptosis and inhibits invasion in U251MG glioblastoma cells. Springerplus 2016;5:235.

-28 Kim HY, Park SJ, Joe EH, Jou I: Raft-mediated Src homology 2 domain-containing proteintyrosine phosphatase 2 (SHP-2) regulation in microglia. J Biol Chem 2006;281:11872-11878.

29 Sturla LM, P. Zinn, and E. Kasper: Suppression of Cellular Senescence in Glioblastoma: Role of Src Homology Domain-Containing Phosphatase 2. Tumor Dormancy, Quiescence, and Senescence. Springer Netherlands 2013;1:707-707.

30 Wang J, Zhang L, Chen G, Zhang J, Li Z, Lu W, Liu M, Pang X: Small molecule 1'-acetoxychavicol acetate suppresses breast tumor metastasis by regulating the SHP-1/STAT3/MMPs signaling pathway. Breast Cancer Res Treat 2014;148:279-289.

-31 Yin S, Wu H, Lv J, Wu X, Zhang Y, Du J, Zhang Y: SHP-1 arrests mouse early embryo development through downregulation of Nanog by dephosphorylation of STAT3. PLoS One 2014;9:e86330. 